5. Which of the following may not result in tracheal collapse?

a. Intrinsic disorder of the trachealis muscle b. Intrinsic disorder of the cartilage matrix

c. Tracheobronchial inflammation

d. Complex disorder of the tracheal architecture

\section{Cough, cough, cough, cough!}

\section{Lynelle Johnson}

Common causes of cough include infectious and inflammatory airway disease in dogs and cats and airway collapse in the dog. Airway collapse can affect the trachea (cervical and/or intrathoracic) or the large airways (bronchomalacia). Small airway collapse can also occur. In general, a dry, honking cough is more typical of airway collapse, while chronic bronchitis more often results in a harsh, hacking cough, however there is a lot of overlap in the type of cough that occurs depending on the amount of secretions present in the airways and the severity of disease. In addition, dogs can suffer from both chronic bronchitis and airway collapse and will generate variable types of cough. Chronic bronchitis and bronchomalacia can be documented in any size or breed of dog, however tracheal collapse is typically a disease of small- or toy-breed dogs. Chronic bronchitis in the cat affects all ages and breeds and, in some cases, can be accompanied by signs of respiratory distress associated with asthma or bronchoconstriction.

The clinical presentation of the dog with tracheal collapse is generally relatively characteristic, although ruling out concurrent infectious or inflammatory airway disease and confirming the site and degree of airway collapse requires fluoroscopy and bronchoscopy. Documenting bronchomalacia is particularly difficult in the absence of advanced diagnostic testing. The diagnosis of chronic bronchitis in the dog and cat is supported by radiographic findings of a bronchial infiltrate, however normal thoracic radiographs can also be encountered and infectious diseases such as Mycoplasma are difficult to rule out with performing specific culture or polymerase chain reaction (PCR) on airway fluid. Empirical therapy with doxycycline can be helpful if these tests are not an option for the client. However, when possible, best practice is to obtain an airway sample to confirm inflammatory disease (generally neutrophilic in the dog, neutrophilic or eosinophilic in the cat). Dogs and cats with bronchitis often require life-long therapy with glucocorticoids. Therefore, verifying a definitive diagnosis is important for long-term management and client communication.

Dogs with airway collapse do not always have concurrent airway inflammation and, in some cases, a steroid trial can result in worsening disease in an affected dog. Presentation of bronchomalacia can be similar to that of bronchitis, with an expiratory push and marked respiratory crackles.
Affected dogs are often overweight and institution of a weight-loss plan can reduce cough. Some dogs can also benefit from administration of extended-release theophylline to improve expiratory effort and lessen the tendency for airway collapse to occur.

\section{KEY LEARNING OBJECTIVES}

- Construct a diagnostic plan to investigate infectious and inflammatory causes of cough

- Detect clues to the differentiation of airway collapse and chronic bronchitis

- Verify the role of glucocorticoids, bronchodilators and weight loss in animals with cough

\section{MULTIPLE CHOICE QUESTIONS}

1. What is the most definitive procedure for diagnosing collapsed trachea?
a. Physical examination
b. Radiography
c. Bronchoscopy
d. Ultrasonography

2. Which of the following would be LEAST likely to cause exacerbation of signs in a dog with tracheal collapse?
a. Endotracheal intubation
b. Thromboembolism
c. Weight gain
d. Chronic bronchitis

3. Which of the following is true?
a. Intrathoracic airway collapse occurs during expiration
b. Only large-breed dogs develop cervical airway collapse
c. Tracheostomy alleviates stridor associated with tracheal collapse
d. Cervical tracheal collapse is associated with expiratory effort

4. Which of the following is not included in management of airway collapse?
a. Theophylline
b. Weight loss
c. Mineralocorticoids
d. Antibiotics for infection

5. Which of the following statement about chronic bronchitis in the dog or cat is true?
a. It is diagnosed by radiographic evidence of a bronchial pattern
b. It is usually accompanied with infection
c. It is responsive to corticosteroid therapy
d. It is caused by dental disease 